\title{
THE SPECTRAL SEQUENCE OF A FINITE GROUP EXTENSION STOPS
}

\author{
BY
}

\section{LEONARD EVENS}

\begin{abstract}
It is proved that if $G$ is a finite group, $H$ a normal subgroup, and $A$ a finitely generated $G$-module, then both the cohomology and homology spectral sequences for the group extension stop in a finite number of stops. A lemma about $\operatorname{Tor}(M, N)$ as a module over $R \otimes S$ is proved. Two spectral sequences of Hochschild and Serre are shown to be the same.
\end{abstract}

1. Introduction. Let $G$ be a finite group, $H$ a normal subgroup, and $A$ a finitely generated $G$-module. We show here that the two spectral sequences

and

$$
H^{p}\left(G / H, H^{q}(H, A)\right) \Rightarrow H^{p+q}(G, A)
$$

$$
H_{p}\left(G / H, H_{q}(H, A)\right) \Rightarrow H_{p+q}(G, A)
$$

stop in a finite number of steps.

Historical note. In [E], I proved a special case of the result for cohomology. Subsequently, I was able to handle the general case along the lines presented here. (At about the same time Atiyah also disposed of the case of cohomology at least for coefficients in $\mathbf{Z}$ by another method.) Since that time $I$ have been asked repeatedly about this fact, and it seems appropriate to provide a published proof. Moreover, there is some added interest to this fact given a recent result of James Blowers which shows that the corresponding spectral sequence for nonnormal subgroup $H$ need not stop. Finally, while I am at it, I take the trouble to deal also with the homology spectral sequence. The situation there is almost, but not quite, dual to that of cohomology.

2. Cohomology. All modules in this section are left modules-but of course this makes no real difference.

THEOREM 1. Let $G$ be a finite group, $H$ a normal subgroup, $A$ a finitely

Received by the editors August 5, 1974.

AMS (MOS) subject classifications (1970). Primary $18 \mathrm{H} 10$. Tor.

Key words and phrases. Lyndon-Hochschild-Serre, spectral sequence, group cohomology, 
generated $\mathrm{Z}(G)$-module. The Lyndon spectral sequence

$$
H^{*}\left(G / H, H^{*}(H, A)\right) \Rightarrow H^{*}(G, A)
$$

stops in a finite number of steps. That is, there exists an integer $r$ such that, for all $p$ and $q, E_{r}^{p, q}=E_{\infty}^{p, q}$.

Proof. The basic idea of the proof is fairly straightforward, but various technical details intervene so as to confuse the matter. Hence, we shall first outline the basic argument and then fill in some details.

Let $E_{r}^{p, q}(A)$ denote the $r$ th term of the spectral sequence for the $G$-module $A$. Consider the subring $R=E_{\infty}^{0, *}(\mathrm{Z})$ of $E_{\infty}(\mathrm{Z}) . R$ is a subring of $E_{2}^{0, *}(\mathrm{Z})=$ $H^{*}(H, \mathrm{Z})^{G}$; hence it is a subring of $H^{*}(H, \mathrm{Z})$. Moreover, by [E, Theorem 7.1], $H^{*}(H, \mathrm{Z})$ and $H^{*}(H, A)$ are finite modules over the noetherian ring $R=E_{\infty}^{0, *}(\mathrm{Z})$. (The latter ring may be identified with $\operatorname{Im}\left\{\right.$ res: $\left.H^{*}(G, \mathrm{Z}) \rightarrow H^{*}(H, \mathrm{Z})\right\}$.) Consequently, by [E, Theorem 6.1], it follows that

(a) $E_{2}(A)=H^{*}\left(G / H, H^{*}(H, A)\right)$ is a noetherian module over $H^{*}(G / H, R)$.

Were the terms $E_{r}(A)$ also modules over $H^{*}(G / H, R)$, the rest of the proof would be clear. (See (f) below.) However, there seems to be no reason why this should be true, hence we make a slight detour.

(b) $H^{*}(G / H, R)$ is noetherian over the subring $H^{*}(G / H, \mathrm{Z}) \otimes R$.

To justify (b), we make use of the universal coefficient theorem exact sequence.

$$
\text { (c) } 0 \rightarrow H^{*}(G / H, \mathrm{Z}) \otimes R \stackrel{\alpha}{\longrightarrow} H^{*}(G, R) \stackrel{\beta}{\longrightarrow} \operatorname{Tor}\left(H^{*}(G / H, \mathrm{Z}), R\right) \rightarrow 0
$$

which is seen to be a sequence of $H^{*}(G / H, \mathrm{Z}) \otimes R$ modules.

(d) $H^{*}(G / H, \mathrm{Z})$ and $R=\operatorname{Im}\left\{\right.$ res: $\left.H^{*}(G, \mathrm{Z}) \rightarrow H^{*}(H, \mathrm{Z})\right\}$ are finitely generated $\mathbf{Z}$ algebras [E, Corollary 6.2]. Hence, so is their tensor product.

(e) It is also true that $\operatorname{Tor}\left(H^{*}(G / H, \mathrm{Z}), R\right)$ is a finite $H^{*}(G / H, \mathrm{Z}) \otimes R$ module.

(This follows from Lemma 2 below which is perhaps of independent interest.) Putting (c), (d), and (e) together establishes (b).

(f) Finally, to complete the proof, notice that the above facts show that $E_{2}(A)$ is a noetherian $H^{*}(G / H, \mathrm{Z}) \otimes R$ module. Also, each $E_{r}(A)$ is a module over this ring, and each $d_{r}$ is a module homomorphism. For each $r$, let $\bar{B}_{r}$ be the pull-back to $E_{2}(A)$ of $\operatorname{Im} d_{r}$. Then $\bar{B}_{2} \subseteq \bar{B}_{3} \subseteq \cdots \subseteq \bar{B}_{r} \subseteq \cdots$ is an ascending chain of $H^{*}(G / H, \mathrm{Z}) \otimes R$ modules which must stop. Suppose $\bar{B}_{r}=\bar{B}_{r+1}=\cdots$. Then $d_{s}=0$ for all $r \geqslant s$, and hence $E_{r}(A)=E_{r+1}(A)=\cdots=E_{\infty}(A)$ is required.

The details. First, we establish a crucial lemma needed in (e).

Lemma 2. Let $A$ and $B$ be rings, $M$ and $N$ (left) noetherian $A$ and $B$ 
modules respectively. Then $\operatorname{Tor}(M, N)$ is a finitely generated $A \otimes B$ module.

Proof. We rely on the treatment of Tor in [Mac, Chapter V, §6]. $\operatorname{Tor}(M, N)$ is generated by elements $\langle m, k, n\rangle$ with $m \in M, n \in N$, and $k \in \mathbf{Z}$ satisfying $k m=k n=0$. Also, if $a \in A$ and $b \in B$, the module structure is defined by

$$
(a \otimes b)\langle m, k, n\rangle=\langle a m, k, b n\rangle .
$$

Let $M_{t}$ and $N_{t}$ be the torsion subgroups of $M$ and $N$ respectively. Clearly, they are submodules and hence are finitely generated over $A$ and $B$ respectively. It follows that $M_{t}$ and $N_{t}$ have finite exponents as abelian groups, and we suppose $\boldsymbol{e}$ is the least common multiple of their exponents. Consider a generator $\langle m, k, n\rangle$ of $\operatorname{Tor}(M, N)$. Let $f=\operatorname{lcm}(o(m), o(n))$. From $k m=k n=0$, it follows that $k=f k^{\prime}$. Thus $\langle m, k, n\rangle=\left\langle m, f k^{\prime}, n\right\rangle=\left\langle m, f, k^{\prime} n\right\rangle$. (See [Mac].) Clearly, $f$ also divides $e$. Hence, we conclude that $\operatorname{Tor}(M, N)$ is generated by elements of the form $\langle m, f, n\rangle$ where $m \in M_{t}, n \in N_{t}$, and $f$ is one of the finite number of divisors of $e$.

Next, for each $f$ dividing $e$, consider the submodules $M_{f}=\{m \mid f m=0\}$ and $N_{f}$. Again, these are finitely generated over $A$ and $B$ respectively. Let $\left\{m_{1}^{(f)}\right.$, $\left.m_{2}^{(f)}, \ldots\right\}$ be a finite set of $A$-module generators for $M_{f}$ and $\left\{n_{1}^{(f)}, n_{2}^{(f)}, \ldots\right\}$ a finite set of $B$-module generators for $N_{f}$. Clearly, the finite set of all $\left\langle m_{i}^{(f)}, f\right.$, $\left.m_{j}^{(f)}\right\rangle$ is a set of $A \otimes B$-module generators for $\operatorname{Tor}(M, N)$.

Next we discuss a whole collection of difficulties arising from the necessity to distinguish a graded (or doubly graded) module from the underlying nongraded module. Related to this is the question of various sign conventions which may or may not be in force depending on the context. We go through the lettered points in the above outline making appropriate comments.

(a) $E_{2}(A)$ is a noetherian $H^{*}(G / H, R)$ module.

(i) $E_{2}(A)=\bigoplus_{p, q} H^{p}\left(G / H, H^{q}(H, A)\right)$ whereas [E, Theorem 6.1] applies to $H^{*}\left(G / H, H^{*}(H, A)\right)$ with $H^{*}(H, A)$ is viewed simply as a (nongraded) $R$-module. Similarly, one may distinguish $H^{*}(G / H, R)$ from $\bigoplus_{p, q \geqslant 0} H^{p}\left(G / H, R^{q}\right)$. Fortunately, in both cases the two objects are the same. To see this simply choose a $G / H$-resolution of $\mathbf{Z}$ which is finitely generated in each degree and note that the required equality already holds on the cochain level.

(ii) $G$ does in fact act trivially on $R=\operatorname{Im}\left\{\right.$ res: $\left.H^{*}(G, \mathrm{Z}) \rightarrow H^{*}(H, \mathrm{Z})\right\}$ as required in Theorem 6.1 of [E].

(iii) The ring structure in $H^{*}(G / H, R)$ and the module structure of $H^{*}\left(G / H, H^{*}(H, A)\right)$ as in Theorem 6.1 are defined directly from the appropriate pairings, while the ring and module structures in $E_{r}(\mathrm{Z})$ and $E_{r}(A)$ respectively $(r \geqslant 2)$ involve a sign convention. That this causes no problems follows from 
LEMmA 3. Let $S=\bigoplus_{p, q \geqslant 0} S^{p, q}$ be a doubly graded ring and $M=$ $\bigoplus_{p, q \geqslant 0} M^{p, q}$ a doubly graded $S$-module. Redefine the ring and module structures by $s^{\circ} m=(-1)^{q p^{\prime}}$ sm where $s$ is of bidegree $(p, q)$ and $m$ is of bidegree $\left(p^{\prime}, q^{\prime}\right)$. Then $M$ is noetherian according to the new structures if and only if it is noetherian according to the original structures.

Proof. Clearly, the bigraded submodules are the same. On the other hand, one knows that a graded module of this kind is noetherian as graded module if and only if it is absolutely noetherian. (The proof is a modification of the proof of the Hilbert basis theorem.)

Note. If you prefer, you can avoid Lemma 3 by replacing $R$ by its subring of elements of even degree. (Careful examination of the proofs in [E] shows that for any group $K, H^{*}(K, \mathrm{Z})$ is a finite module over the noetherian commutative subring of elements of even degree.)

(c) (For information on the universal coefficient theorem see [Mac, Chapter V, $\S \S 10,11]$ )

(iv) Let $X \rightarrow \mathrm{Z}$ be a convenient $G / H$-free resolution, e.g. the standard resolution. Then $C=\operatorname{Hom}_{G / H}(X, Z)$ is a differential graded algebra, finitely generated and $Z$-free in each degree. In particular,

$$
\operatorname{Hom}_{G / H}(X, R) \cong \operatorname{Hom}_{G / H}(X, \mathrm{Z}) \otimes R=C \otimes R
$$

and this is an isomorphism of differential graded algebras. (For the moment, we ignore the fact that $R$ is a graded ring itself.) The universal coefficient theorem provides an exact sequence,

$$
0 \rightarrow H^{*}(C) \otimes R \stackrel{\alpha}{\longrightarrow} H^{*}(C \otimes R) \stackrel{\beta}{\longrightarrow} \operatorname{Tor}\left(H^{*}(C), R\right) \rightarrow 0
$$

which translates into the sequence (c). (The reader is referred to [Mac] for the definitions of $\alpha$ and $\beta$.) $\alpha$ is in fact a ring homomorphism making the middle term an $H^{*}(C) \otimes R$ module as required.

(v) To verify that $\beta$ is a module homomorphism, show its "inverse" $\gamma: \operatorname{Tor}\left(H^{*}(C), R\right) \rightarrow H^{*}(C \otimes R) / \operatorname{Im} \alpha$ [Mac, Chapter V, Proposition 10.6] is a module homomorphism. Indeed, $\gamma$ is defined as follows: Given $\langle\bar{u}, k, r\rangle$, we have $k u=d v$; then $\gamma\langle\bar{u}, k, r\rangle$ is represented by $(-1)^{n+1} v \otimes r$ where $n=\operatorname{deg} u$. A routine calculation using formula (1) yields the desired result.

(d) $H^{*}(G / H, \mathrm{Z})$ and $R$ are in fact anticommutative graded rings. (According to (iii) above, we may include the sign convention in defining the tensor algebraor not-as convenient.) To show that $H^{*}(G / H, \mathrm{Z}) \otimes R$ is noetherian, one has two choices. One can extend the Hilbert basis theorem-which is not too difficult. Alternatively, one can look at the commutative subrings of $H^{*}(G / H, \mathrm{Z})$ and $R$ of 
elements of even degree. These are also finitely generated $\mathbf{Z}$ algebras, and $H^{*}(G / H, \mathrm{Z}) \otimes R$ is a finite module over their tensor product.

(f) Module structure in the spectral sequence.

(vi) For each $r \geqslant 2$, we have ring homomorphisms

$$
R=E_{\infty}^{0, *}(\mathrm{Z}) \rightarrow E_{r}^{0, *}(\mathrm{Z}) \subseteq E_{r}(\mathrm{Z})
$$

and

$$
H^{*}(G / H, \mathrm{Z})=E_{2}^{*, 0}(\mathrm{Z}) \rightarrow E_{r}^{*, 0}(\mathrm{Z}) \subseteq E_{r}(\mathrm{Z})
$$

and the images in $E_{r}(\mathrm{Z})$ (anti) commute. This yields a ring homomorphism $H^{*}(G / H, \mathrm{Z}) \otimes R \rightarrow E_{r}(\mathrm{Z})$ where the tensor product is of graded rings. Since, for each $r \geqslant 2, E_{r}(A)$ is an $E_{r}(Z)$-module in a way consistent with the spectral sequence structure, we get the required $H^{*}(G / H, \mathrm{Z}) \otimes R$-module structure in the spectral sequence.

(vii) For $r=2$, one may check that-except for the sign conventionthe ring structure in $H^{*}(G / H, \mathrm{Z}) \otimes R$ and accompanying module structure in $E_{2}(A)=H^{*}\left(G / H, H^{*}(H, A)\right)$ is the same as that involved in the above noetherian discussion. Because of remark (iii), we can ignore the difference.

3. Homology. In principle, one could derive the analogue to Theorem 1 for homology by dualizing the entire theory including the results of [E]. It does not seem, however, that the appropriate results for homology are completely dual to those for cohomology. Hence, rather than trying to develop a theory of conoetherian comodules, we shall use duality in a somewhat less monolithic way and simply reduce the homology case to that for cohomology.

THEOREM 4. Let $G$ be a finite group, $H$ a normal subgroup, and $A$ a finitely generated $G$-module. Then the homology spectral sequence

$$
H_{*}\left(G / H, H_{*}(H, A)\right) \Rightarrow H_{*}(G, A)
$$

stops in a finite number of steps, i.e., there is an integer $r$ such that $E_{p, q}^{r}(A)=$ $E_{p, q}^{\infty}(A)$ for all $p, q$.

Proof. By duality arguments, we may reduce the problem to showing that the cohomology sequence

$$
H^{*}\left(G / H, H^{*}(H, \hat{A})\right) \Rightarrow H^{*}(G, \hat{A})
$$

stops. $(A=\operatorname{Hom}(A, Q / Z)$.) To be more explicit, suppose $A$ is a $G$-module, $X$ is a $G$-projective resolution of $\mathbf{Z}$, and $Y$ is a $G / H$-projective resolution of $\mathbf{Z}$. (We suppose all modules are left modules, but again this makes no essential difference.) Then the homology spectral sequence may be derived from the double complex 
$Y \otimes_{G / H}\left(X \otimes_{H} A\right)$. Moreover, we have a natural isomorphism

$$
\operatorname{Hom}\left(Y \otimes_{G / H}\left(X \otimes_{H} A\right), \mathrm{Q} / \mathrm{Z}\right) \cong \operatorname{Hom}_{G / H}\left(Y, \operatorname{Hom}_{H}(X, \hat{A})\right)
$$

provided by

$$
F \leftrightarrow f \text { where } F(y \otimes(x \otimes a))=(f(y)(x))(a) .
$$

(Recall $\bar{g}(x \otimes a)=g x \otimes g a,(\bar{g} f)(x)=g f\left(g^{-1} x\right)$, etc.) The right-hand side is a double complex defining the cohomology spectral sequence, and since $\operatorname{Hom}(-, Q / Z)$ is exact, we see that the cohomology spectral sequence is obtained from the homology spectral sequence by applying that functor. On the other hand, for $r \geqslant 2$ and $p$ and $q$ not both zero, the groups $E_{p, q}^{r}(A)$ are all finite. Thus $d_{p, q}^{r}: E_{p, q}^{r}(A) \rightarrow E_{p-r, q+r-1}^{r}$ is trivial if and only if its dual $d_{r}^{p-r, q+r-1}$ : $E_{r}^{p-r, q+r-1}(\hat{A}) \rightarrow E_{r}^{p, q}(\hat{A})$ is trivial.

We now show that the cohomology sequence for $\hat{A}$ does in fact stop. To do so we must refine the proof of Theorem 1 so as to derive the conclusion with a somewhat weaker hypothesis. Write $M=\hat{A}$. Put $H^{+}(H, M)=\bigoplus_{q>0} H^{q}(M)$. To apply the argument in (f), it suffices to show that $H^{*}\left(G / H, H^{+}(H, M)\right)$ is a finitely generated $H^{*}(G / H, Z) \otimes R$ module and that $H^{+}\left(G / H, M^{H}\right)$ is a finitely generated $H^{*}(G / H, Z)$-module. (Split up $\bar{B}_{r}$ appropriately.) To prove the first contention, it is enough to show $H^{+}(H, M)$ is finitely generated over $R$ which we do below. Since $\left.M^{H}=\widehat{A /[H, A]}\right)$, substantially the same argument takes care of the second contention.

Since $A$ is finitely generated, we have the short exact sequence of $G$-modules

$$
0 \rightarrow A_{t} \rightarrow A \rightarrow F=A / A_{t} \rightarrow 0
$$

where $A_{t}$ is the torsion subgroup of $A$ and $F$ is $Z$-free of finite rank.

Consider the dual sequence

$$
0 \rightarrow \hat{F} \rightarrow \hat{A} \rightarrow \hat{A}_{t} \rightarrow 0 .
$$

Clearly it suffices to show that the ends of the exact sequence

$$
H^{+}(H, \hat{F}) \rightarrow H^{+}(H, \hat{A}) \rightarrow H^{+}(H, \hat{A})
$$

are $R$-noetherian.

$\hat{A}_{t}$ is finite so that there is no trouble with the right end. To deal with the left end, note first that $\hat{F}$ is a divisible abelian group-even a direct sum of copies of $\mathbf{Q} / \mathbf{Z}$. Thus the sequence

$$
0 \rightarrow(\hat{F})_{h} \rightarrow \hat{F} \stackrel{h}{\longrightarrow} \hat{F} \rightarrow 0
$$

is exact. Since $H^{+}(H, \hat{F}) \stackrel{h}{\longrightarrow} H^{+}(H, \hat{F})$ is trivial, $H^{+}\left(H,(\hat{F})_{h}\right) \rightarrow H^{+}(H, \hat{F})$ is an epimorphism. On the other hand $(\hat{F})_{h}$ is also a finite group and its cohomology is noetherian. 
4. Historical note. The basic facts concerning the spectral sequence of a group extension were described in [HS]. There, the authors derived two spectral sequences with $E_{2}$ term $H^{*}\left(G / H, H^{*}(H, A)\right)$ and ending in $H^{*}(G, A)$. They did not, however, show the two sequences are the same. Their first sequence is easily seen to be that described above arising from the double complex $\operatorname{Hom}_{G / H}\left(Y, \operatorname{Hom}_{H}(X, A)\right)$ when $Y$ is taken to be the standard resolution for $G / H$ and $X$ the standard resolution for $G$. Their second sequence involves a special filtration of $C(G, A)=\operatorname{Hom}_{G}\left(X_{N}, A\right)$ where $X_{N}$ is the normalized standard resolution. It is only for the second sequence that they construct a product structure.

In view of these remarks, there would seem to be a gap in the argument presented here. There are two ways to fill this gap. First, one can introduce the product structure in the first spectral sequence. The author has done this in unpublished work. It is a tedious but straightforward exercise in homological algebra. (What is needed, clearly, is a systematic presentation of the spectral sequence-as a functor-in terms of general resolutions.)

Secondly, one can show that the two spectral sequences are the same. We do this here in order to settle what is a minor but annoying point.

We shall demonstrate that the spectral sequence arising from the double complex $\operatorname{Hom}_{G / H}\left(Y_{N}, \operatorname{Hom}_{H}\left(X_{N}, A\right)\right)$ is the same as that constructed in Chapter II ("The direct method") of [HS]. We may replace the standard resolutions by the normalized standard resolutions by virtue of the unpublished theory alluded to above. (The reader will readily accept that, in the double complex theory, one has freedom to vary the resolutions $X$ and $Y$ as necessary without changing the spectral sequence.)

Adapting the notation of [HS], we define $F^{p} C^{p+q}(G, A)$ to be the collection of normalized cochains $g\left(z_{1}, z_{2}, \ldots, z_{p+q}\right)$ which vanish if at least $q+1$ arguments belong to $H$. The spectral sequence of this filtration yields the "second"-cup product consistent-spectral sequence of [HS] . Moreover, let ${ }^{*} F^{p} C^{p+q}(G, A)$ consist of all cochains $g\left(x_{1}, x_{2}, \ldots, x_{q}, y_{1}, \ldots, y_{p}\right)$ which depend only on the cosets of the last $p$ arguments. Then ${ }^{*} F^{p} C \subseteq F^{p} C$ and [HS] shows that the inclusion induces an isomorphism of spectral sequences for $r \geqslant 1$ (Proposition 1 , p. 119).

$$
\begin{aligned}
& \text { For } g \in{ }^{*} F^{p} C^{p+q} \text {, define }{ }_{p} g \in C^{p}\left(G / H, C^{q}(H, A)\right) \text { by } \\
& \qquad p g\left(\bar{y}_{1}, \ldots, \bar{y}_{p}\right)\left(u_{1}, \ldots, u_{q}\right)=g\left(u_{1}, u_{2}, \ldots, u_{q}, y_{1}, \ldots, y_{p}\right)
\end{aligned}
$$

for $u_{1}, u_{2}, \ldots, u_{p} \in H$. ( $y_{i}$ represents $\bar{y}_{i}$.) It is shown in [HS] that ${ }_{p+1}(d g)=$ $\left.d^{\prime \prime}{ }_{p} g\right)$ where $d^{\prime \prime}$ is the differential in the complex $C(H, A)$. Hence $g \mapsto{ }_{p} g$ yields a map ${ }^{*} E_{1}^{p, q} \rightarrow C^{p}\left(G / H, H^{q}(H, A)\right)$ which [HS] shows is an isomorphism (Theorem 1, p. 121). 
We shall define a map of complexes

$$
\Psi: \operatorname{Hom}_{G / H}\left(Y_{N}, \operatorname{Hom}_{H}\left(X_{N}, A\right)\right) \rightarrow C(G, A)
$$

which is filtration preserving. Moreover, the composite map $f \mapsto{ }_{p}(\Psi(f))$ will be shown to induce an isomorphism

$$
\operatorname{Hom}_{G / H}\left(\left(Y_{N}\right)_{p}, H^{q}(H, A)\right) \rightarrow C^{p}\left(G / H, H^{q}(H, A)\right) .
$$

Thus, on the $E_{1}$ level, $\Psi$ induces an isomorphism and hence, it induces an isomorphism of spectral sequences as required.

To define $\Psi$, we first remind the reader about the structure of $Y_{N}$ and $X_{N}$. $\left(Y_{N}\right)_{p}$ is the free $\mathrm{Z}(G / H)$ module with basis consisting of all $\left[\bar{y}_{1}, \ldots, \bar{y}_{p}\right]$ with each $\bar{y}_{i} \in G / H$-except that $\left[\bar{y}_{1}, \ldots, \bar{y}_{p}\right]=0$ if some $\bar{y}_{i}=1$. The differential is given by the usual formula. $\left(X_{N}\right)_{q}$-as an $H$-module-is the free $\mathrm{Z}(H)$-module with basis consisting of all $y\left[x_{1}, x_{2}, \ldots, x_{q}\right]$ where each $x_{i} \in G$, no $x_{i}=1$, and $y$ is in a predetermined set of coset representatives of $H$ in $G$. Again, we exclude from the basis any $y\left[x_{1}, \ldots, x_{q}\right]$ with some $x_{i}=1$ by setting these equal to zero. It follows that the differentials in the double complex $\operatorname{Hom}_{G / H}\left(Y_{N}, \operatorname{Hom}_{H}\left(X_{N}, A\right)\right)$ are given as follows. For $f$ of bidegree $(p, q)$, we have

$$
\begin{aligned}
\left(d_{I} f\right)\left(\left[\bar{z}, \bar{y}_{1}, \ldots, \bar{y}_{p}\right]\right)\left(y\left[x_{1}, \ldots, x_{q}\right]\right) \\
=z f\left(\left[\bar{y}_{1}, \ldots, \bar{y}_{p}\right]\right)\left(z^{-1} y\left[x_{1}, \ldots, x_{q}\right]\right) \\
\quad-f\left(\left[\bar{z}, \bar{y}_{1}, \ldots, \bar{y}_{p-1}\right]\right)\left(y\left[x_{1}, \ldots, x_{q}\right]\right) \\
\quad+\cdots+(-1)^{p+1} f\left(\left[\bar{z}, \bar{y}_{1}, \ldots, \bar{y}_{p-1}\right]\right)\left(y\left[x_{1}, \ldots, x_{q}\right]\right)
\end{aligned}
$$

and

$$
\begin{aligned}
&\left(d_{I I} f\right)\left(\left[\bar{y}_{1}, \ldots, \bar{y}_{p}\right]\right)\left(y\left[x_{1}, \ldots, x_{q}, z\right]\right) \\
&=(-1)^{p}\left\{f\left(\left[\bar{y}_{1}, \ldots, \bar{y}_{p}\right]\right)\left(y x_{1}\left[x_{2}, \ldots, x_{q}, z\right]\right)\right. \\
&-\cdots+(-1)^{q} f\left(\left[\bar{y}_{1}, \ldots, \bar{y}_{p}\right]\right)\left(y\left[x_{1}, \ldots, x_{q} z\right]\right) \\
&\left.\quad+(-1)^{q+1} f\left(\left[\bar{y}_{1}, \ldots, \bar{y}_{p}\right]\right)\left(y\left[x_{1}, \ldots, x_{q}\right]\right)\right\} .
\end{aligned}
$$

The total differential is $d=d_{I}+d_{I I}$. by

For $f \in \operatorname{Hom}_{G / H}\left(\left(Y_{N}\right)_{p}, \operatorname{Hom}_{H}\left(\left(X_{N}\right)_{q}, A\right)\right)$, define $g=\Psi(f) \in C^{p+q}(G, A)$

$$
\begin{aligned}
& g\left(x_{1}, \ldots, x_{q}, y_{1}, \ldots, y_{p}\right) \\
& \quad=(-1)^{p q} x_{1} x_{2} \cdots x_{q} f\left(\left[\bar{y}_{1}, \ldots, \bar{y}_{p}\right]\right)\left(\left(x_{1} \cdots x_{q}\right)^{-1}\left[x_{1}, \ldots, x_{q}\right]\right)
\end{aligned}
$$


Extend $\Psi$ by linearity. Note that $g \in{ }^{*} F^{p} C \subseteq F^{p} C$; hence $\Psi$ preserves filtrations. Also, a routine calculation demonstrates

$$
d(\Psi(f))=\Psi(d f)
$$

Consider next the map $\Psi^{\prime}: f \mapsto{ }_{p} g$ where $f$ and $g$ are as above. We have, for $u_{1}, u_{2}, \ldots, u_{q} \in H$,

$$
\begin{aligned}
& \left.p^{g\left(\bar{y}_{1}\right.}, \ldots, \bar{y}_{p}\right)\left(u_{1}, \ldots, u_{q}\right) \\
& \quad \begin{aligned}
(7) \quad & (-1)^{p q} u_{1} u_{2} \cdots u_{q} f\left(\left[\bar{y}_{1}, \ldots, \bar{y}_{p}\right]\right)\left(\left(u_{1} u_{2} \cdots u_{q}\right)^{-1}\left[u_{1}, \ldots, u_{q}\right]\right) \\
& =(-1)^{p q} f\left(\left[\bar{y}_{1}, \ldots, \bar{y}_{p}\right]\right)\left(\left[u_{1}, u_{2}, \ldots, u_{q}\right]\right) .
\end{aligned}
\end{aligned}
$$

(The last equality holds since $f\left(\left[y_{1}, \ldots, y_{q}\right]\right) \in \operatorname{Hom}_{H}\left(X_{N}, A\right)$.) Thus, except for sign, $\Psi^{\prime}(f)\left(\left[\bar{y}_{1}, \ldots, \bar{y}_{p}\right]\right)$ is just the restriction of $f\left(\left[y_{1}, \ldots, y_{p}\right]\right)$ to basis elements $\left[u_{1}, \ldots, u_{q}\right]$ with $u_{1}, u_{2}, \ldots, u_{q} \in H$.

The induced map

$$
\operatorname{Hom}_{G / H}\left(\left(Y_{N}\right)_{p}, H^{q}(H, A)\right) \rightarrow C^{p}\left(G / H, H^{q}(H, A)\right)
$$

is-except for sign-the identity. Since (8) factors through

$$
E_{1}^{p, q}=\operatorname{Hom}_{G / H}\left(\left(Y_{N}\right)_{p}, H^{q}(H, A)\right) \rightarrow{ }^{*} E_{1}^{p, q}
$$

we have finished.

\section{REFERENCES}

[E] L. Evens, The cohomology ring of a finite group, Trans. Amer. Math. Soc. 101 (1961), 224-239. MR 25 \#1191.

[Mac] S. Mac Lane, Homology, Die Grundlehren der math. Wissenschaften, Band 114, Academic Press, New York; Springer-Verlag, Berlin, 1963. MR 28 \#122.

[HS] G. Hochschild and J.-P. Serre, Cohomology of group extensions, Trans. Amer. Math. Soc. 74 (1953), 110-135. MR 14, 619.

DEPARTMENT OF MATHEMATICS, NORTHWESTERN UNIVERSITY, EVANSTON, ILLINOIS 60201 